\title{
Service Level Driven Stock Allocation: A Model Based Enterprise Information System
}

\author{
Felix S. Wriggers ${ }^{1}$, Matthias Schmidt ${ }^{1}$, Rouven Nickel $^{2}$ and Peter Nyhuis ${ }^{1}$ \\ ${ }^{1}$ Institute of Production Systems and Logistics, Gottfried Wilhelm Leibniz Universität \\ Hannover, Hannover, Germany wriggers@ifa.uni-hannover.de schmidt@ifa.uni-hannover.de \\ ${ }^{2}$ Institute of Integrated Production Hannover, Hannover, Germany nickel@iph-hannover.de
}

\begin{abstract}
The conflict between the objectives for logistic efficiency and storage costs largely influences inventory management. The positioning in this tension field poses a challenge to decision makers. The Logistic Storage Analysis (LSA) is an industry proven control model used to dimension stock within this field of tension. To this end, the LSA offers a variety of tools, derived from Logistic Operating Curves, for aggregating and visualizing data and is thus an ideal foundation and starting point for the Enterprise Information System (EIS) currently being developed.
\end{abstract}

Keywords: EIS for manufacturing sector, Logistic operations, Logistic operation curves, Stock allocation, Supply chain management, Supply chain planning and execution

\section{INTRODUCTION}

Like the price and quality of the products, the logistic efficiency of industrial firms has become an important criterion for purchasing during the last two decades. The trend to consider parameters for logistic efficiency as well as the qualities of a company or a product has established itself in both national and international markets [1-2]. The logistic objectives, shorter delivery times and high due date reliability have thus taken on added significance with this development [3-5].

In order to meet these demands, the central logistic challenge in the controlling of procurement, storage and distribution processes turns out to be ensuring the availability of required parts for the customer at the planned time.

The availability of parts is ensured in operating plants through inventory management in that from a logistic viewpoint, the stock in the procurement or distribution stores has to be adequately dimensioned. In addition to availability, costs related to maintaining stock have to be considered in order to economically dimension it [6]. Here, we see the classical Dilemma of Inventory Management: A balance has to be found between a high availability of parts on the one hand and low stock costs on the other hand $[2,7-8]$.

As the primary goal, the firm's profitability is defined in accordance with the economical goals [7]. Consequently, efficiently managing costs and minimizing running costs are basic prerequisites for remaining competitive. 
Stock dimensioning plays a major role here, as the costs related to extensive capital tie-up significantly influences the production costs. If some of the capital tie-up can be resolved, the freed capital might be directly utilized for investments and thus for generating future cash flows.

Nevertheless, from both the procurement and distributions sides, industrial firms' storage strategies are frequently not oriented on demand. Parts which are often required regularly and in large numbers are often out of stock, whereas those sporadically required are often stored in large amounts and for long periods. Thus for example stock accounts for ca. $22 \%$ of the balance sheet total in the German automobile ancillary industry [9].

In order to support companies in improving their inventory management it is necessary to develop a simple, field oriented Enterprise Information System. Such a system should offer decision making support for the Logistic Positioning of stock levels within the field of tension between the need for greater storage efficiency (higher service level, lower delivery delay) and low stock costs (low stock level). At the same time this system has to provide decision makers with up to the minute information including operating figures and when necessary, visualize them [10]. Building on Throughput Diagrams, Storage Operating Curves and Ranking Lists the Logistic Storage Analysis presents an ideal basis for such a system.

\section{THE LOGISTIC STORAGE ANALYSIS}

The Logistic Storage Analysis is a method with which a firm can analyze its stock and uncover potential for a greater service level. It is theoretically well established in numerous publications $[7-8,11]$ and has proven to be a useful instrument for analysis in a large number of applications including those in the field [e.g. 12-13]. Currently the Logistic Storage Analysis is being expanded to implement interdependencies of service levels and a different service level definition.

The Logistic Storage Analysis consists of a variety of methods (cf. Figure 1) such as the Storage Throughput Diagram, Storage Operating Curves and Ranking Lists. These can be used to transparently represent storages processes as well cause and effect relations, in addition to indicating problem areas and identifying potentials [8].

During a Logistic Storage Analysis the required modeling methods are linked together with one another, whereby scenarios with different boundary conditions are created through systematically varying the parameters which determine the stock level.

Taking into consideration different boundary conditions makes it possible to derive laws as well as to estimate the consequences of prospective measures identify problem articles and define objectives. Dimensioning the required safety stock level for example, leads to an increased reliability in storage processes and thus to an improved service level.

Experiences in practical applications have shown that the results can only be sustained and the potential can only be used over the long term, when a regular storage analysis is supported through the development of a logistic objective control. 
This is due to the continual changes in a company and thus also to the shifting logistic environment, which requires the stock to be continuously monitored and adjusted.
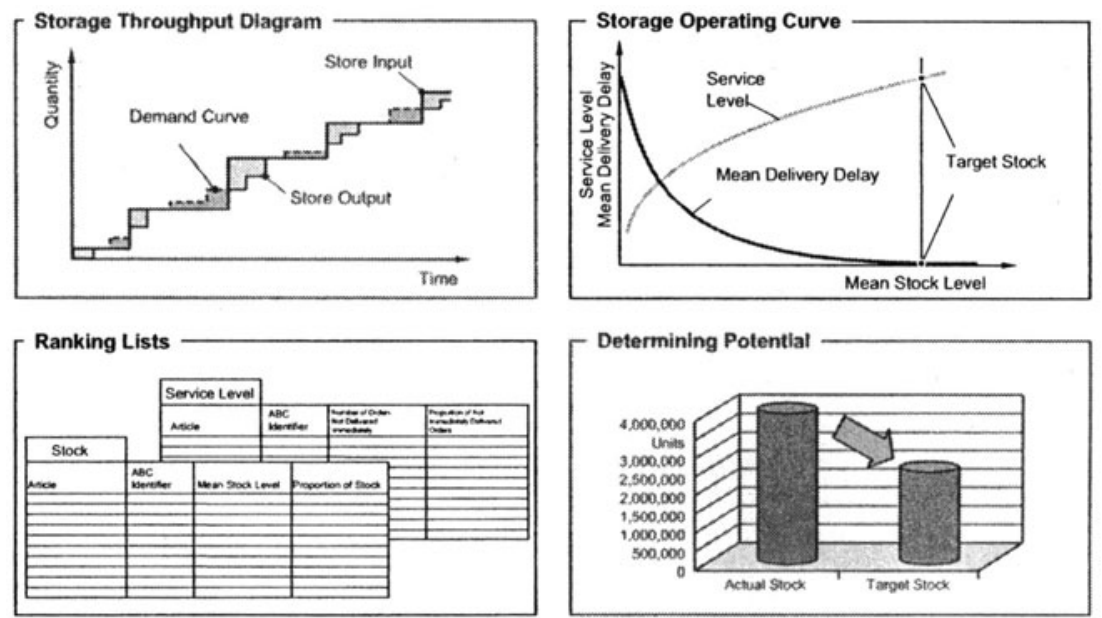

Figure 1. Logistic Storage Analysis Methods

Applying the Logistic Storage Analysis in the industrial field demonstrated a number of possibilities for improving it, particularly with regards to implementing inventory analyses and controls. On the one hand it became clear that the required data was not easily drawn from the system and that an intensive validation process had to be undertaken. On the other hand, there were problems in a few cases when converting it to a continuous control: The effort necessary to generate important figures from the data could not be justified and the problem could not be clearly visualized near to real time.

The idea to develop an Enterprise Information System, which offers users support in the decision making process thus emerged. After an initial storage analysis and dimensioning, this system should contribute to the continual analysis and control of the stores. At the same time this system should take into consideration two important further developments of the Logistic Storage Analysis: the Allocation Diagram and the unweighted Service Level Operating Curves.

\section{EXPANDING THE LOGISTIC STORAGE ANALYSIS}

\subsection{The Unweighted Service Level}

The service level is a common operating figure in the industrial field and offers an approach for evaluating the trade-off between stock costs and stock-out costs [14]. 
Generally, the service level, which can be either weighted or unweighted, describes the degree to which the demands on the stores are fulfilled. Up until now, the Logistic Storage Analysis only took into consideration the weighted service level.

Since the unweighted service level is however more frequently applied than the weighted service level in the industry, expanding the Logistic Storage Analysis to include the unweighted Service Level Operating Curve offers a better possibility to meet practical demands [7].

The weighted service level $\left(S E R L_{w}\right)$ is determined from the ratio of punctually withdrawn demanded parts to the total demanded quantity. The demanded orders are thus weighted with their respective amounts.

Through this weighting with the demand lot size, the significance and influence of the different order sizes is reflected on the logistic efficiency of a stocking echelon. Based on the $\mathrm{C}_{\text {norm }}$ function, LUTZ developed a weighted Service Level Operating Curve, which expresses the cause and effect relations between the Work in Process and weighted service level [7].

The unweighted service level $\left(S E R L_{u w}\right)$ corresponds to the ratio of demands that are met both time wise and quantity wise to the total demand.

Just as the weighted Service Level Operating Curve represents the service level over the mean stock level per article, so does the newly developed unweighted Service Level Operating Curve. Similarly, the $\mathrm{C}_{\text {norm }}$ function is also employed as a basis.

The correlation between the two service levels, allow inferences to be drawn about a store's distribution behavior. Thus when $\mathrm{SERL}_{\mathrm{w}}>\mathrm{SERL}_{\mathrm{uw}}$ it indicates that few large demands were punctually served, and that many smaller demands also faced delays. In contrast, the reverse situation, where $S E R L_{u w}>S E R L_{w}$, indicates that many small demands are punctually served and only a few large orders are delayed.

With regards to the criteria relevant to making decisions, a method for choosing the Logistic Operating Curve best suited for the respective problem was developed. This method also permits for the first time, inferences to be made from a stores' target service level about the article service level. In doing so, the degree of correlation (if applicable) between individual articles in the store is considered.

Further, factors influencing the $C_{\text {norm }}$ function's shape were identified and structured according to their effects. Future work to determine an equation for the shape depending on the identified influencing factors is conducted at present.

\subsection{The Allocation Diagram}

When the customer is not separated from the production through a store, then the customer's desired service level is primarily dependent on the logistic synchronization between the firm's manufacturing and assembly areas. In order for a company to have a high logistic efficiency it is a prerequisite that all of the manufacturing orders required for an assembly order be completely available and allocated from the store for the planned assembly start date.

When single part orders are late being filled it frequently leads to delays in the corresponding assembly orders and thus has a direct, negative impact on the completion date. If a completion date in contrast is met earlier than planned or a part 
of it is retrieved too early and the assembly starts only at the planned date, the corresponding part has to be buffered. Costs then accumulate for the resulting Work in Process buffer through the tied up capital costs, handling and required floor space.

The Allocation Diagram developed in order to analyze the scheduling situation for the order network and to improve the analysis of adjusting the manufacturing and assembly processes [15-16]. The goal of this diagram is to visualize and precisely synchronize the provisions from the store and/or the preceding manufacturing.

An Allocation Diagram consists of two curves. The first curve represents the connection of the points in time at which the first position of an assembly order is allocated. The Completion Curve in contrast delivers the completion time of all an assembly order's positions, accumulated and sorted according to the point in time of the last allocated manufacturing order (Figure 2).

The area which is created between the two curves corresponds to the Work in Process of the partially allocated manufacturing orders. In order to be able to transparently represent the scheduling situation of different assembly orders, the respective planned requirement dates are normalized, that is set to zero. According to NICKEL the Allocation Diagram can be utilized for depicting the loss of value due to badly adjusted processes also [16].

So that the Work in Process buffers and scheduling deviations can be reduced in the output, a simultaneous and punctual allocation should be strived for. With reference to the diagram, that means that the two curves should approximate one another as closely as possible in the area before the normalized requirement date i.e. the point of origin.

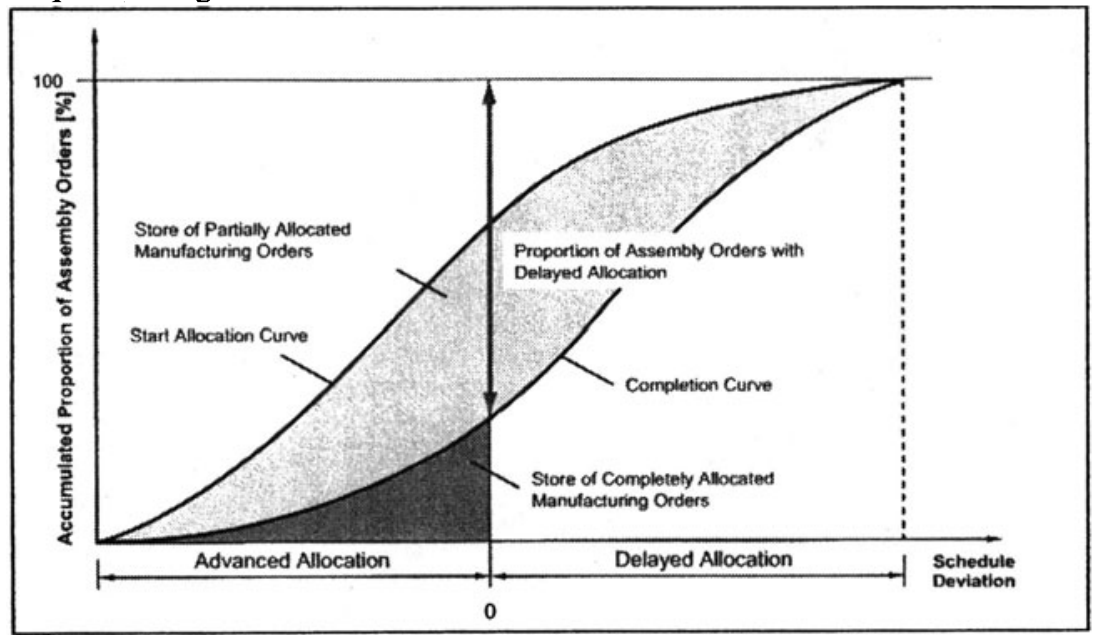

Figure 2. The Allocation Diagram

The Allocation Diagram might be deployed for diverse purposes. Both analysis of the current state of allocation processes and derivation of measures to improve upon the current state are furthered [16]. With the Allocation Diagram it is possible to indicate potential for improving the delivery reliability and reducing unnecessary 
stock, as well as to derive the measures required for developing these identified potentials [17].

Measures such as sequencing according to the slack rule, can further the punctual allocation. In order to support the simultaneous completion of the orders though, a reactive schedule oriented release is required which is based on realistic, order specific, planned throughput times. Dates determined through backwards scheduling have to be consistently used. Should however, an individual order's completion be delayed in an order network; it is advisable to shift the planned completion date for all other manufacturing orders in order to avoid an unplanned Work in Process buffer.

So that orders are punctually allocated, the flexibility of the capacities can be increased in the manufacturing area and/or the allocation of the manufacturing capacities can be distributed according to demands. Further measures, which promote the punctual and simultaneous availability of manufacturing orders include scheduling controls in both the manufacturing and assembly areas, as well as developing products that enable the complexity of an order's work content structure to be reduced.

\section{DEVELOPMENT OF THE ENTERPRISE INFORMATION SYSTEM}

By expanding the Logistic Storage Analysis to include the unweighted Service Level Operating Curves and the Allocation Diagram, the field of application for the Logistic Storage Analysis can also be extended. With the assistance of a further method, which can be applied in order to choose the most suitable Service Level Operating Curve, the direct back-coupling of a target service level (for the entire inventory) can occur for the first time based on the target stock levels of individual articles. In this way, it is possible to optimally structure the stores in the entire inventory with regards to costs (Figure 3 ).

Experiences during the development of the LogiAs Enterprise Information System for the make process, demonstrated that modeling with methods from the Logistic Operating Curves presented an almost ideal basis for such a system with regards to the ratio of required effort and illustrative quality. NICKEL AND VOGEL describe this EIS (LogiAs) and the applicability of the Logistic Operating Curve Theory in great detail [18]. LogiAs applies the Logistic Operating Curve Theory in order to control near to real time production processes. In doing so a variety of variables and Logistic Operating Curves are available for the user, which are supplemented through additional instruments (e.g., portfolios, scenarios, and ranking lists). These variables and Logistic Operating Curves are determined near to real time, based on the validated data from the data warehouse and are visualized according to the users wish.

It can thus be presumed that the above mentioned aspects of the expanded Logistic Storage Analysis form a very good basis for an Enterprise Information System. Therefore the Enterprise Information System currently under development at the Institute for Production Systems and Logistics will utilize the Logistic Storage Analysis and the Methods included therein. 


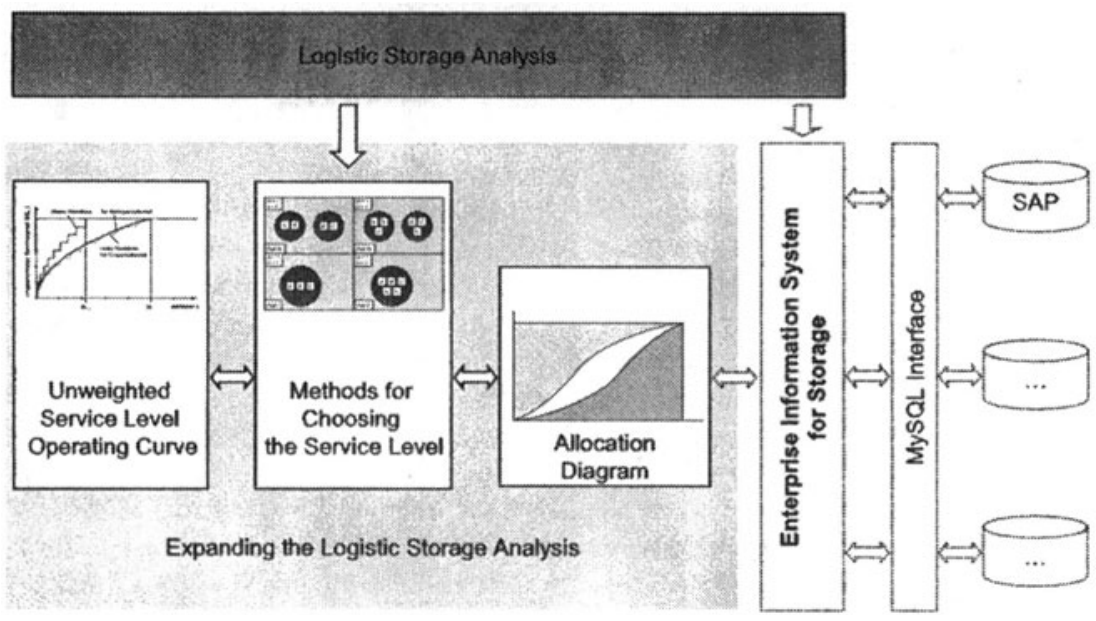

Figure 3. Locating the Enterprise Information System for Storage within the Field of Research on Storage Analysis

Building on real data gathered in an ample investigation period of the near past and validated for accuracy and correctness the System may then be used for several purposes in the field of stock dimensioning. An overview of the Logistic Stock Analysis capabilities is given in Figure 4.
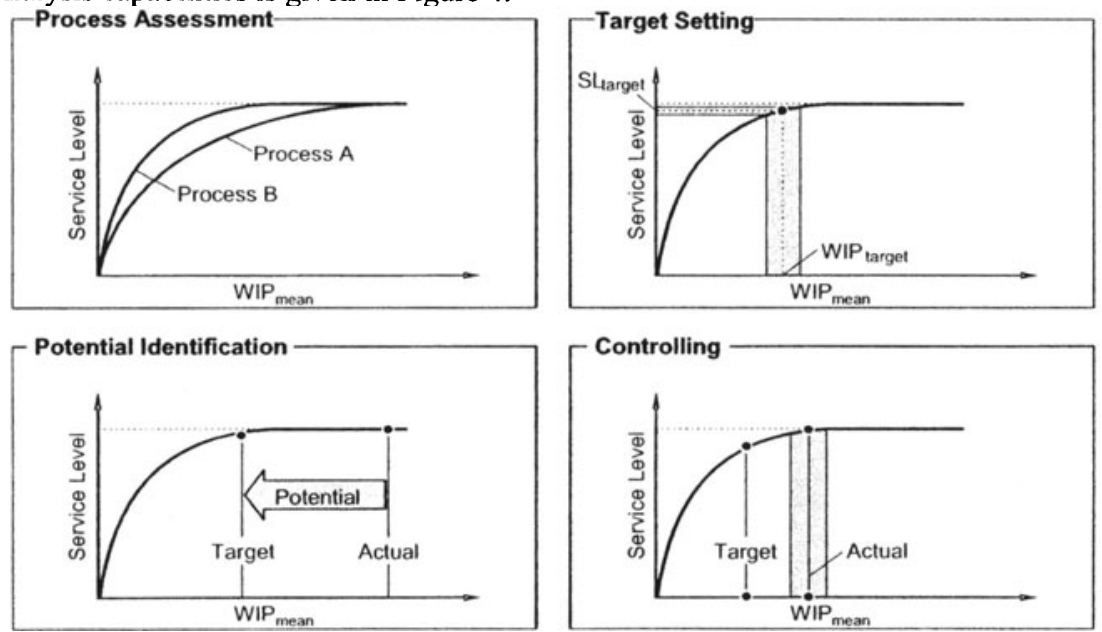

Figure 4. Capabilities of the Logistic Storage Analysis

On field of application for the Enterprise Information System is the assessment of alternative processes. Since the input parameters of Logistic Operating Curves are the parameters of upstream processes, alternative shapes of the curve result subject to 
their efficiency. For instance if the upstream process is a sourcing process involving suppliers, the suppliers can be assessed by means of Logistic Operating Curves. For each supplier individual Operating Curves can be generated establishing the option to evaluate the supplier quality. The supplier to be favored is the one sporting the best Operating Curve.

Service Level Operating Curves may also be generated if the upstream process is in-house. For this purpose the process's target variances can be used since the shape of the Operating Curve depends on them. Varying of these parameters poses the chance to study effects of process improvements (e.g. an improvement of on time delivery). Resulting Work in Process levels and Service Levels can be compared and thus the potential for optimization can be analyzed.

Besides for process assessment Service Level Operating Curves and respectively the Enterprise Information Systems can be utilized in setting targets. Service Level Operating Curves have to fulfil two functions for this purpose: Both the process of target setting and of target assessment. By using the Operating Curves it can be determined if aimed at targets are consistent with Service Levels and Work in Process. If the targeted Work in Process is not part of the Service Level Operating Curve the targeted Service Level is not obtainable for the targeted Work in Process for existing boundary conditions. Therefore measures have to be derived, to change the Operating Curves shape.

An essential field of application for Service Level Operating Curves is the determination of Work in Process necessary to achieve targeted Service Levels. The Enterprise Information System will be able to achieve this goal by calculating the Work in Process corresponding with the targeted Service Level. This Work in Process can then be used as target Work in Process. If the targeted Service Level is given with a range of tolerance, a likewise range of tolerance can be calculated for Work in Process. This range of tolerance can then be utilized for definition of action limits.

Service Level Operating Curves can be used to depict actual and target operating points. They are thus suited for a logistical Work in Process and Warehouse controlling. The Enterprise Information System thus aids a quick visualization of actual and target Work in Process and Service Levels. Considering influences fields of action can be derived. Effects of derived measures on the processes may also be evaluated. Thus an assessment as well as a concerted application of measures is aided by the Enterprise Information System.

Last but not least the potential inherent in the process can be discerned by comparing actual and target Work in Process. Expanding this comparison to all articles in stock results in the cumulative Work in Process adjustment necessary for one warehouse. This adjustment might be a reduction as well as an increase in overall Work in Process. It is imperative to check for further measures if the necessity for increase in Work in Process is determined.

\section{ACKNOWLEDGEMENTS}

The authors would like to thank the German Research Foundation (DFG), which financially supports the expansion of the Logistic Storage Analysis within the context 
of the project "Developing a Method for Increasing Part Availability in Assembly Areas through Service Level Oriented Inventory Dimensioning".

\section{REFERENCES}

1. T. Tracht and S. Reinsch, Erfolgsfaktor Logistikqualität: Vorgehen, Methoden und Werkzeuge zur Verbesserung der Logistikleistung, 2nd Edition, eds. H.P. Wiendahl (Springer: Berlin, 2002), pp.1-7.

2. H. Wildemann, Logistik-Check - Identifikation und Erschließung von Logistikpotenzialen, 5th Edition (TCW: München, 2007).

3. K.K.B. Hon, Performance and Evaluation of Manufacturing Systems, Annals of the CIRP. Volume 55, Number 2, (2005).

4. P. Nyhuis, F. Wriggers, and A. Fischer, Knowledge Enterprise: Intelligent Strategies in Product Design, Manufacturing, and Management, in Proc. of The International Federation of Information Processing (IFIP), Volume 207, eds. K. Wang, G. Kovacs, M. Wozny, and M. Fang (Springer: Boston, 2006).

5. B. Enslow, Best Practices in International Logistic (Aberdeen Group: Boston, 2006).

6. J. Gläßner, Modellgestütztes Controlling der beschaffungslogistischen Prozesskette, Fortschritt-Berichte VDI, Series 2, Number 337 (VDI: Düsseldorf, 1995).

7. S. Lutz, Kennliniengestütztes Lagermanagement, Fortschritt-Berichte VDI, Series 13, Number 53 (VDI: Düsseldorf, 2002).

8. P. Nyhuis and H.P. Wiendahl, Logistische Kennlinien - Grundlagen, Werkzeuge und Anwendungen, 2nd Edition (Springer: Berlin, 2003).

9. K. Gerhardt and C. Sarantidis, Automobilzulieferer-Bericht zur Branche, IKB Information Dezember 2003 (IKB: Düsseldorf, 2003).

10. M. Shah and M. Littlefield, There is No Execution without Integration - MES Adoption Drives Performance (Aberdeen Group: Boston, 2007).

11. S. Lutz and H. Lödding, and H.P. Wiendahl, Logistics-oriented inventory analysis, International Journal of Production Economics. Volume 85, Number 6, pp.217-231, (2003).

12. M. Vogel, M. Schmidt, D. Emminger, and A. Mix, Entwicklung eines Beschaffungskonzepts im Maschinenbau, Supply Chain Management. Volume 6, Number 2, pp.39-44, (2006).

13. F.S. Wriggers, T. Busse, and M. Schmidt, Logistische Lageranalyse und Methodenvalidierung im Elektronikwerk Amberg, Industrie Management. Volume 23, Number 5, publication pending, (2007).

14. J. Alscher, H. Schneider, Zur Interdependenz von Fehlmengenkosten und servicegrad, Kostenrechnungspraxis. Volume 6, pp.257-271, (1982).

15. P. Nyhuis, R. Nickel, and T. Busse, Logistisches Controlling der Materialverfügbarkeit mit Bereitstellungsdiagrammen, Zeitschrift für wirtschaftlichen Fabrikbetrieb. Volume 101, Number 5, pp.265-268, (2006).

16. R. Nickel, Logistische Modelle für die Montage (PZH: Hannover, 2007), publication pending.

17. P. Nyhuis, R. Nickel, and D.Grabe, Synchronisationspotenziale in der Logistik identifizieren und bewerten, PPS-Management. Volume 12, Number 2, pp.66-69, (2007).

18. R. Nickel and M. Vogel, Entwicklung eines Assistenzsystems für das Produktionscontrolling, Industrie Management. Volume 22, Number 4, pp.61-64, (2006). 